\title{
Pancreatic abscess due to Salmonella typhi
}

\author{
Pradeep Garg and Sunil Parashar
}

Department of Surgery, Medical College \& Hospital, Rohtak, Haryana, India Summary: Isolated involvement of the pancreas in Salmonella typhi bacteraemia is rare. A case of
pancreatic abscess due to $S$. typhi is reported which was managed conservatively.

\section{Introduction}

Salmonella infection occurs in 5 different clinical forms, gastroenteritis, enteric fever, bacteraemia, chronic carrier state and localization at one or more sites. Localization in the pancreas is rarely seen and when it does has mostly required surgical intervention. We report a case of Salmonella typhi pancreatitis progressing to abscess, managed conservatively.

\section{Case report}

A 20 year old male was admitted with fever and epigastric pain for 8 days and vomiting of 3 days duration. On examination he was toxic with pulse $130 / \mathrm{min}$, temperature $38.9^{\circ} \mathrm{C}$ and mild jaundice. Abdominal examination revealed tenderness and rigidity in the upper abdomen. The white cell count was $12.8 \times 10^{9} / 1$ with polymorphs $81 \%$, blood urea $16.6 \mathrm{mmol} / 1$. Erect abdominal X-ray film showed no gas under the diaphragm. He was given gentamicin, ampicillin and metronidazole. Next morning a vague tender lump was felt strengthening the clinical suspicion of acute pancreatitis with possibly a pseudocyst. Serum amylase was $375 \mathrm{~S}$. units (normal up to $200 \mathrm{~S}$. units). An urgent computed tomographic (CT) scan showed a pancreatic abscess which was enhanced after giving i.v. contrast.

His condition further deteriorated over the next 24 hours. The three antibiotics were replaced by ciprofloxacillin infusion $200 \mathrm{mg} 12$ hourly. Blood culture showed $S$. typhi. Ciprofloxacillin too was replaced by intravenous chloramphenicol and simultaneously blood for Widal test was also sent which was reported to be strongly positive. Within

Correspondence: P. Garg, M.S.(Surgery), 687/27, Opp. Medical Crossing, Model Town, Rohtak-124001, Haryana, India.

Accepted: 21 August 1991
$24 \mathrm{~h}$ of starting chloramphenicol the patient started to improve and 2 weeks after admission repeat CT scan showed a normal pancreas.

\section{Discussion}

Localized salmonella infection of the pancreas is usually the result of salmonella bacteraemia caused by $S$. choleraesuis but may also occur after gastroenteritis by $S$. typhimurium and enteric fever by $S$. typhi. ${ }^{1}$ Once pancreatitis occurs it is likely to form a pancreatic abscess. Pancreatic pseudocyst may occasionally be infected by $S$. typhi. ${ }^{2} S$. typhi is known to localize in injured or damaged tissue or in sites of malignancy. ${ }^{3}$ The route of infection in pancreatic abscess has not been clearly demonstrated. Possible pathways may be infected bile reaching the pancreas by the pancreatic duct, haematogenous spread from a distant site and lymphatic spread from the intestinal tract. ${ }^{4}$

There have been few published reports of localized pancreatic involvement in typhoid fever. Kune and Coster ${ }^{5}$ reported a case of pancreatic abscess due to $S$. typhi who had gallstones; $S$. typhi was isolated from the necrotic pancreatic tissue and gallstones. Russell et $a l^{6}$ have reported acute pancreatitis as a complication of typhoid fever and two cases of chronic pancreatitis by $S$. typhi were reported by Koshi. ${ }^{2}$ One of these required distal pancreatectomy while the other required only drainage of a pseudocyst. The first case of pancreatic abscess due to $S$. typhimurium was reported by Strand and Sanders. ${ }^{4}$ This case had associated gallstones and chronic cholecystitis but culture of gall bladder wall, bile and gallstones showed no growth. Cohen et al. ${ }^{1}$ have also reported a similar case but without associated gall bladder involvement.

In the present case, since the patient showed clinical and biochemical recovery with conservative treatment, the tentative plan of CT-guided fine 
needle aspiration cytology of the pancreatic abscess and subsequently culturing was abandoned. Serial urine and stool cultures in the present case failed to grow S. typhi.

\section{References}

1. Cohen, J.I., Bartlett, J.A. \& Correy, G.R. Extraintestinal manifestations of salmonella infection. Medicine 1987, 66: 349-388.

2. Koshi, G. Uncommon manifestations of salmonella infections. Ind $J$ Med Res 1976, 64: 314-321.

3. Saphra, I. \& Winter, J.W. Clinical manifestations of salmonellosis in man - an evaluation of 7,779 human infections identified at the New York salmonella centre. $N$ Engl $J$ Med 1957, 256: 1128-1134.

4. Strand, C.L. \& Sanders, S.L. Salmonella typhimurium pancreatic abscess - report of a case. Am Surg 1978, 44: 174-176.

5. Kune, G.A. \& Coster, D. Typhoid pancreatic abscess. Med J Aust 1972, 1: 417-418.

6. Russell, I., Forgars, P. \& Geraci, J. Pancreatitis complicating typhoid fever. JAMA 1976, 235: 753. 